\title{
Effectiveness of the Freestyle Libre Flash Glucose Monitoring System on Diabetes Distress Among Individuals with Type 1 Diabetes: A Prospective Study
}

\author{
Ayman A. Al Hayek · Asirvatham A. Robert · Mohamed A. Al Dawish
}

Received: December 24, 2019 / Published online: March 7, 2020

(C) The Author(s) 2020

\section{ABSTRACT}

Aim: To study and explore the intervention of the flash glucose monitoring system (FGMS) on diabetes-related distress (DRD) in children and adolescents with type 1 diabetes (T1D).

Methods: A 12-week prospective study was performed from March 2019 to July 2019 involving 187 children and adolescents (age range $13-19$ years; $56.7 \%$ female) with T1D who were self-testing their glucose levels using the conventional fingerprick method. At the time of the baseline visit, FGMS sensors were fixed by a trained diabetes educator onto each patient in the study population. A trained interviewer also administered the 28-item T1-Diabetes Distress Scale (T1-DDS) questionnaire to each participant at the baseline visit and again after 12 weeks to determine the T1-DDS score.

Results: Comparison of the baseline (fingerprick) data with data collected at 12 weeks after the patients had switched to the FGMS revealed

Enhanced Digital Features To view enhanced digital features for this article go to https://doi.org/10.6084/ m9.figshare. 11866053 . a significant decrease in the subdomains of the T1-DDS as follows: powerlessness $(p=0.0001)$; management distress $(p=0.0001)$; hypoglycemia distress $(p=0.0001)$; negative social perceptions $(p=0.0001)$; eating $(p=0.0001)$; physician distress $(p=0.0001)$; friend/family distress $(p=0.0001)$; and total T1-DDS score $(p=0.0001)$. Similarly, analysis of the data revealed that there was also a substantial drop from baseline to 12 weeks after initiation of the intervention in the clinical variables assessed, such as glycosylated hemoglobin; specifically, there was a considerable decrease after 12 weeks in the frequency of hypoglycemia. Interestingly, the frequency of glucose monitoring also showed an upswing among users of the FGMS. Conclusion: The outcomes of this study clearly demonstrate that once the patients had been switched from the fingerprick method to FGMS, the DRD and related clinical parameters showed remarkable improvement. However, further studies are necessary to determine whether the continued and consistent use of the FGMS will achieve better results.

Keywords: Diabetes mellitus; Diabetes therapy; Type 1 diabetes

A. A. Al Hayek ( $₫)$ · A. A. Robert · M. A. Al Dawish

Department of Endocrinology and Diabetes,

Diabetes Treatment Center, Prince Sultan Military

Medical City, Riyadh, Saudi Arabia

e-mail: ayman.alhayek@yahoo.com 


\section{Key Summary Points}

Why carry out this study?

To the best of our knowledge, there has been no research to date on the effects of the flash glucose monitoring system (FGMS) on diabetes-related distress (DRD) among young patients with type 1 diabetes (T1D) in the Arab region, especially Saudi Arabia.

The aim of this study was, therefore, to explore the effect of the FGMS on seven different subdomains of the T1-Diabetes Distress Scale (T1-DDS) among young individuals with T1D.

\section{What was learned from the study?}

The switch from glucose monitoring using the conventional fingerprick method to that using the FGMS resulted in a substantial drop in the values of the clinical variables assessed, such as glycosylated hemoglobin, after 12 weeks.

A considerable decrease was also noted after 12 weeks in the frequency of hypoglycemia.

Interestingly, the frequency of glucose monitoring also showed an upswing among users of the FGMS after 12 weeks.

The outcomes clearly demonstrate that once the patients had switched from the fingerprick method to the FGMS, DRD and related clinical parameters showed remarkable improvements.

\section{INTRODUCTION}

Multiple medical and social myths have been linked to type 1 diabetes (T1D), particularly diabetes-related distress (DRD) [1]. However, it is clearly evident that high levels of DRD are common among individuals with T1D (prevalence 18-35\%; 18-month incidence $38-48 \%$ ) and that these persist over time; in addition, high DRD levels are markedly recognizable from clinical depression in terms of their associations with glycemic control and disease management $[2,3]$. Many patients with diabetes report frustration with the burdens of disease management, as well as worries, fears, and concerns about the possible emergence of complications, irregular blood glucose levels, hypoglycemic episodes, and feelings of "diabetes burnout" [3-5].

Although flexible insulin regimens enable patients to be more successful in achieving treatment goals, these regimens also put patients under greater pressure $[4,5]$. It is significant that many adult patients with T1D experience disease-related psychological difficulties that frequently go unnoticed and, consequently, are either untreated or poorly managed. Such poor treatment strategies can prevent patients from improving their individual care in terms of accomplishing several selfcare tasks and reaching good glucose control [4-6]. The clinical importance of recognizing and treating T1D-linked psychological distress and its outcomes as a crucial health problem is emphasized by the understanding that T1D ranks among the major factors that contribute towards both depression and distress in a high percentage of adults who suffer from this disease $[7,8]$. DRD has been operationalized in many ways, and several steps to deal with it are currently in practice. Clarity in its assessment is an important requirement from both the scientific and clinical perspectives [9].

Until very recently, the majority of studies conducted on DRD have involved adult patients with type 2 diabetes (T2D). However, the results of clinical research on DRD in adults with T2D have not been compared with those from similar studies in adults with T1D, who exhibit very different disease-related issues and experiences [10]. Adult patients with T1D manifest symptoms of simple phobias and general anxiety and depression as well as DRD at twice the frequency seen in the general population. Specifically, anxiety and depression in adults with T1D have also been related to poor glycemic control, 
which in turn exerts very detrimental effects on daily functioning and quality of life [11].

The flash glucose monitoring system (FGMS) is a recent approach to glucose monitoring that can replace fingerprick testing by offering a more convenient option when intensive glucose monitoring is required. This new monitoring tool represents an innovative method for easily monitoring the interstitial fluid glucose level in diabetic patients. The FGMS allows patients to monitor their glucose levels with no discomfort at any time of the day, thereby avoiding even the pain of fingerpricks. In addition, both individual blood glucose readings (as registered using glucometers) and glucose level trends (as determined using continuous glucose monitoring) can be easily recorded [12-15]. Several recent studies have shown that FGMS is precise, with high patient tolerance and that it can reliably decrease glucose variability and lengthen the time in range; it is also uncomplicated in terms of wearing and usage [16-19]. However, to the best of our knowledge, there has to date been no research on the effects of FGMS and its influence on DRD among young patients with T1D in the Arab region, especially Saudi Arabia. Therefore, the aim of this study was to to explore the influence exerted by the FGMS on the scores of different subdomains of the 28-item T1-Diabetes Distress Scale (T1-DDS) questionnaire among young patients with T1D.

\section{METHODS}

\section{Study Design and Sampling}

For this prospective study, we selected a convenience sample of 187 children and adolescents with T1D (age range 13-19 years) who used the conventional fingerpricking method to self-test their glucose levels and whose T1D was managed at the Diabetes Treatment Center, Prince Sultan Military Medical City (PSMMC), Riyadh, Saudi Arabia between March 2019 to July 2019. All procedures followed were in accordance with the Helsinki Declaration of 1964, as revised in 2013. The Research and Ethics Committee of Prince Sultan Military
Medical City, Riyadh, Saudi Arabia, approved the study protocol. All participants in the study or their parents/caregivers received instructions regarding their roles and provided signed informed consent prior to recruitment.

Prior to recruitment to the study, the participants had no experience with using the FGMS and had received insulin treatment by continuous subcutaneous insulin infusion (CSII) or multiple-dose injections (MDIs) for a minimum of 6 months prior to recruitment. The exclusion criteria prohibited these patients from registering to use another interstitial glucose monitoring system while participating in the present study and during the 6 months prior to inclusion in the study. Other exclusion criteria were: (1) dermatological disorders or alterations at the sensor application site within 6 months of the study; (2) severe or unstable medical conditions; (3) severe hypoglycemia necessitating third-party assistance; (4) diabetic ketoacidosis; (5) hyperosmolar-hyperglycemic state; and (6) pregnancy. All participants reserved the unconditional or absolute "right" to withdraw from the study at any time point in the study, and neither reason nor prior notice for withdrawal was needed.

The first study visit was considered to be baseline. At baseline, the following details were recorded on a standardized case record form: (1) demographic data, (2) clinical features, and (3) treatment history (administration of MDIs and insulin pump).

\section{Education on the FGMS}

Prior to starting the study, each participant and his/her parents/guardians received comprehensive education and written instructions on the use of the FGMS. These instructions included requesting the patients/parents/guardians to hold the reader $1-4 \mathrm{~cm}$ away from the sensor for $1 \mathrm{~s}$; teaching them that the sensor can be scanned through clothing; and showing them how to change the sensor once every 14 days [20]. Demonstrations were given on how blood glucose levels could be confirmed using a capillary measurement in the event of imminent and/or suspected hypoglycemia, rapidly changing 
glucose levels, or when their symptoms failed to match the reading on the FGMS, by using the in-built blood glucose meter reader.

All the participants were also given contact information of the educator, who could be accessed at any point of time during this study. When the educational session was completed, a trained diabetes educator competent in applying the FreeStyle Libre (Abbott Laboratories, Chicago, Ill, USA) sensors and in training the participants in the procedure fixed the sensor on the back of the upper arm of each participant. Six sensors were attached to each participant; two extra sensors were also attached in the event of sensor detachment. When the study was completed, all data recorded from the sensors were computed, and the corresponding ambulatory glucose profiles (AGPs) were generated in order to determine the total number of scans done during the 90-day study period. For data interpretation, the mean number of scans per day was taken into consideration.

\section{Glycosylated Hemoglobin and Hypoglycemia}

The American Diabetes Association has endorsed the measurement of the gycosylated hemoglobin (HbA1c) level as an accurate diagnostic test for diabetes. An HbA1c level of $<7 \%$ indicates good blood glucose level control. Hypoglycemia is defined as a confirmed blood glucose value of $\leq 70 \mathrm{mg} / \mathrm{dL}$. The HbA1c levels in this study were analyzed twice during the study, once at baseline and once at 12 weeks after the FGMS testing was initiated, by COBAS INTEGRA 400 plus/800 analyzers (Roche Diagnostics, Indianapolis, IN, USA) at the central laboratory of PSMMC.

At baseline, clinical data for the preceding 4 weeks, including frequency of conventional self-monitoring of blood glucose (SMBG) by fingerprick and frequency of hypoglycemia, were collected using the FreeStyle Optium Neo ${ }^{\circledR}$ blood glucose meter (Abbott Laboratories) equipped with the FreeStyle Auto-Assist Neo ${ }^{\circledR}$ software prior to the commencement study, At 12 weeks after baseline, the flash data were collected from the FGMS sensors and computed to generate the respective AGPs so as to determine the total number of scans conducted and the frequency of hypoglycemia during the study period. Study participants were instructed to take capillary measurements if they experienced impending or possible hypoglycemic events, glycemic variability, or inconsistent symptoms using the in-built blood glucose meter reader.

\section{T1-DDS Score}

The DRD data were collected at baseline and end of the study (i.e., 12 weeks). The T1-DDS (hereafter referred to as the DDS) is a reliable and widely used method which gives an accurate assessment of diabetes distress in patients with T1D [10]. A trained interviewer administered (via interview) the DDS questionnaire to each patient.

The DDS comprises seven concepts of health wherein five questions (\#5, 9, 13, 21, and 25) deal with powerlessness (a broad sense of discouragement regarding diabetes); four questions (\#1, 8, 12, and 28) relate to management distress (disappointment with self-care efforts); four questions (\#3, 15, 22, and 27) relate to hypoglycemia distress (concerns regarding hypoglycemic events); four questions (\#4, 10, 19 , and 24) deal with negative social perceptions (worry about other people's possible negative judgments); three questions (\#2, 16, and 23) deal with eating distress (anxiety that eating is out of control); four questions (\#7, 14, 18, and 26) address physician distress (disappointment with the healthcare professionals currently managing their treatment); and four questions $(\# 6,11,17$, and 20) relate to friend/family distress (a perception of too much focus on diabetes among their loved ones). Scoring is as follows: for each question, 1 represents not a problem; 2, a slight problem; 3, a moderate problem; 4, a somewhat serious problem; 5 , a serious problem; and 6 , a very serious problem. A higher overall score indicates a higher distress level with the diabetes (higher DRD) [10]. 


\section{Statistical Analysis}

The data were analyzed using Microsoft Excel 2013 (Microsoft Corp., Redwood, WA, USA) and the Statistical Package for Social Sciences

Table 1 Baseline characteristics of the study population $(n=187)$

\begin{tabular}{l} 
Variable(s) \\
\hline Age (years) \\
$<15$ \\
$>15$
\end{tabular}

Frequency, $n$

(\%)

Gender

Female

$106(56.7)$

Male

$81(43.3)$

Body mass index $\left(\mathrm{kg} / \mathrm{m}^{2}\right)$

$<25$

$\geq 25$

$84(44.9)$

Duration of diabetes (years)

$\leq 5$

$>5$

Treatment

Multiple-dose injection

$129(69)$

Continuous subcutaneous insulin

$58(31)$ infusion

HbAlc (\%)

$$
<7
$$

$>7$

Frequency of glucose monitoring (per day)

$\begin{array}{ll}1 & 54(28.9) \\ 2 & 67(35.8) \\ 3 & 48(25.7) \\ >3 & 18(9.6)\end{array}$

Values in table are presented as the number (frequency) with the percentage in parenthesis

HbAlc Glycosylated hemoglobin (version 22; IBM-SPSS Inc., Armonk, NY, USA). The two-tailed paired $t$ test was performed to identify the differences among the sets in terms of the different time points (baseline vs. 12 weeks). The Pearson correlation coefficient was used to determine the total number of scans performed in the total DDD score, as well as the HbA1c levels and hypoglycemia value. A $p<0.05$ was considered to be statistically significant.

\section{RESULTS}

The clinical and demographic traits of the study samples are shown in Table 1. Mean age of the participants was 15.3 years, and $56.7 \%$ of the study sample were female. The majority of the study population were older than 15 years, and $64.7 \%$ had been diagnosed with diabetes for $\leq 5$ years. A total of $49 \%$ of participants had a body mass index $\geq 25 \mathrm{~kg} / \mathrm{m}^{2}$, and $69 \%$ were on MDI treatment. The majority $(88.2 \%)$ had high HbA1c values.

The effectiveness of the FGMS on each clinical variable assessed is shown in Table 2 . Significant drops were evident in the clinical variables of $\mathrm{HbA1c}$ and hypoglycemia. There was an increase in the frequency of glucose monitoring (Table 2).

Table 2 Comparison of clinical parameters at baseline and after 12 weeks of intervention

\begin{tabular}{lll}
\hline Variable(s) & $\begin{array}{l}\text { Baseline (before } \\
\text { FGMS } \\
\text { intervention) }\end{array}$ & $\begin{array}{l}\text { 12 weeks after } \\
\text { initiation of } \\
\text { FGMS }\end{array}$ \\
\hline HbA1c (\%) & $8.2 \pm 2.3$ & $7.9 \pm 2.1$ \\
$\begin{array}{l}\text { Frequency of } \\
\text { glucose } \\
\text { monitoring }\end{array}$ & $2.1 \pm 1.2$ & $6.7 \pm 1.7$ \\
$\begin{array}{l}\text { Hypoglycemic } \\
\text { events (per } \\
\text { month) }\end{array}$ & $6.3 \pm 1.7$ & $4.7 \pm 1.9$ \\
\hline
\end{tabular}

FGMS Flash glucose monitoring system 

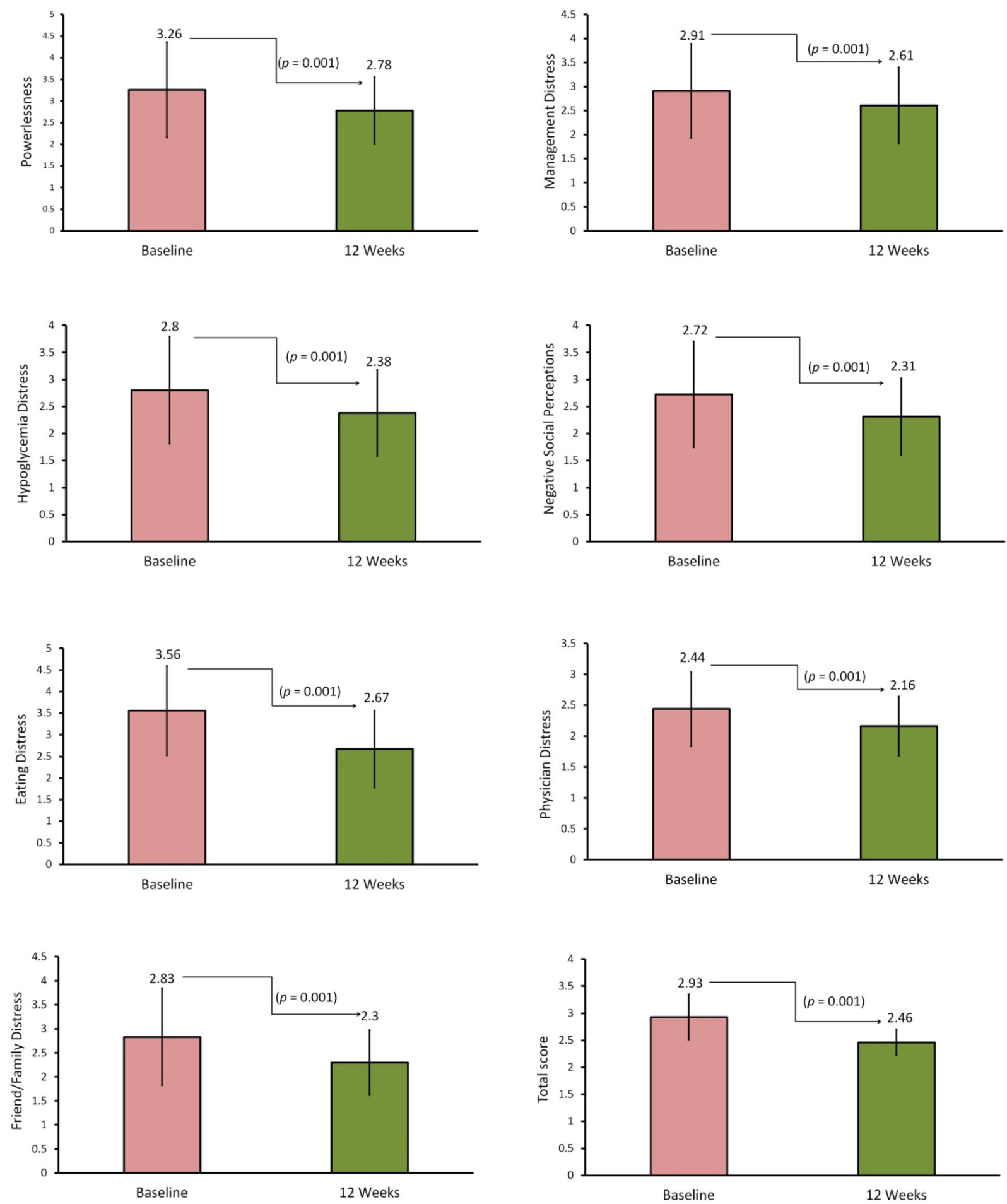

Fig. 1 Effectiveness of the flash glucose monitoring system on T1-Diabetes Distress Scale (T1-DDS) subdomains of diabetes-related distress (comparison of baseline vs. 12 weeks after intervention)

Compared to the DDS scores at baseline (glucose monitoring by fingerprick), the scores for the DDS subdomains of powerlessness $(p=0.0001)$, management distress $(p=0.0001)$, hypoglycemia distress $(p=0.0001)$, negative social perceptions $(p=0.0001)$, eating 


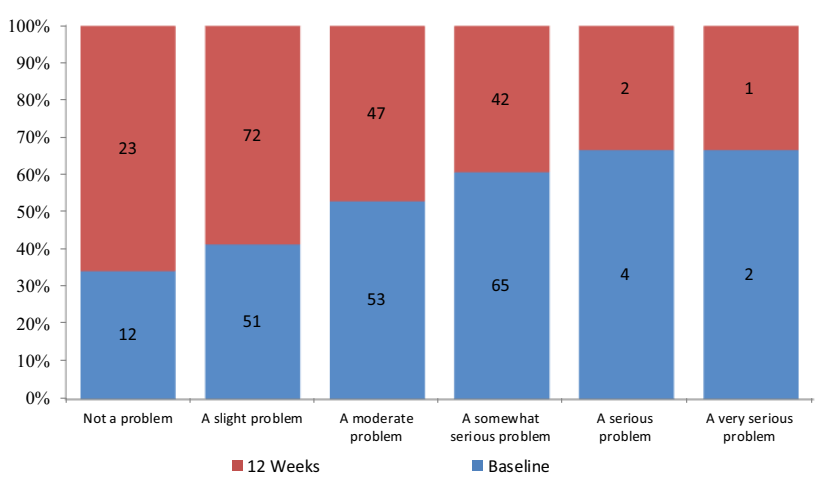

Fig. 2 Patient classification according to the severity of diabetes-related distress

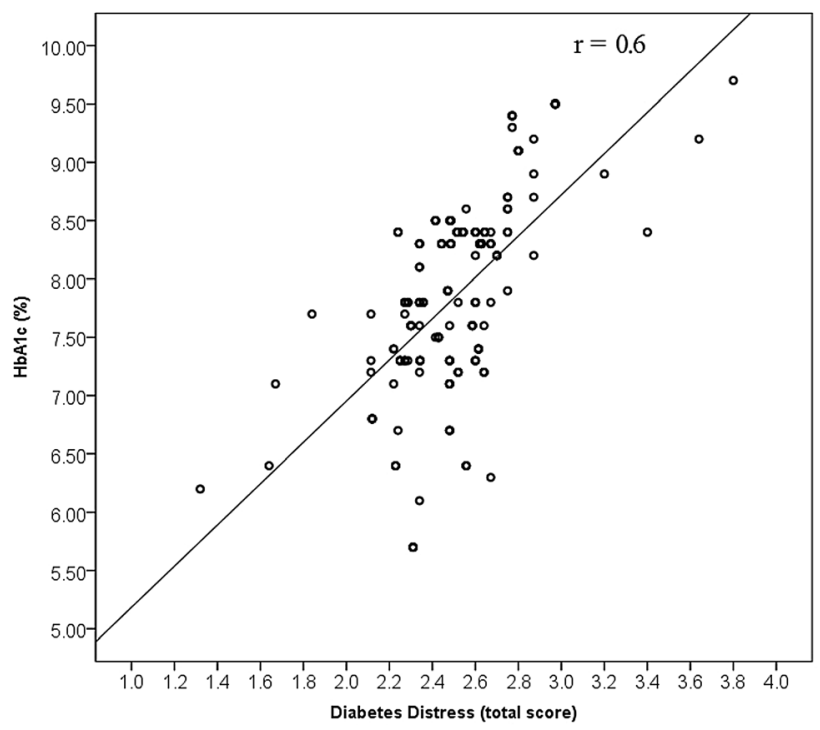

Fig. 3 Pearson correlation of glycated hemoglobin $(H b A l c)$ level and total T1-DDS scores

$(p=0.0001)$, physician distress $(p=0.0001)$, friend/family distress $(p=0.0001)$, and total DDS score $(p=0.0001)$ were substantially lower at 12 weeks after the patients were switched from glucose monitoring by fingerprick to glucose monitoring by FGMS (Fig. 1). Similarly, 12 weeks after the patients had switched to FGMS, HbA1c was notably lower compared to baseline. There was also a significant lowering of hypoglycemia at 12 weeks compared to baseline and, interestingly, the frequency of glucose monitoring had increased at 12 weeks among the FGMS users.

Classification of the patients based on the severity of the DDS scale score (not a problem, a slight problem, a moderate problem, a somewhat serious problem, a serious problem, a very serious problem) is shown in Fig. 2 . Following the intervention with the FGMS, a significant number of patients showed a shift to scores indicating not a problem and a slight problem from scores indicating a somewhat serious problem, a serious problem, and a very serious problem.

A positive correlation was noted in $\mathrm{HbA1c}$ level $(r=0.6)$, hypoglycemia $(r=-0.35)$, and total DDS score and alterations in the DDS 'inducing' changes in the HbA1C and hypoglycemia, or vice versa (Fig. 3 ). 


\section{DISCUSSION}

The aim of the present study was to assess the impact of the FGMS on seven distinct subdomains of the DDS among young patients with T1D. The score for the DDS subdomain "powerlessness" at 12 weeks after the patients started using the FGMS was clearly lower than that obtained at baseline using the fingerprick method ( $p=0.0001)$. The findings of this study concur with the outcomes of several earlier studies which reported that a large percentage of patients with diabetes experienced feelings of frustration due to the pressure of disease management and anxieties, fears, and discouragement as well as concerns regarding the potential appearance of complications, irregular blood glucose values, hypoglycemic episodes, and feelings of diabetes-related stress [21, 22].

The results of many studies have revealed that in order to maintain optimal daily blood glucose control, children and adolescents with T1D need to adopt a complex self-regulatory behavior each and every day that involves the completion of multiple daily adherence behaviors, such as daily checks for blood glucose levels and insulin dosing, that may be tedious and cause a variety of management distress-related issues [23, 24]. Self-control and psychosocial complications are inversely related, with the main psychosocial complications being anxiety and depression. Compared to adults, adolescents are 2.3-fold more liable to experience such psychosocial problems [23, 24]. We noted that management distress among the T1D patients was high at baseline and that a notable reduction in this stress had occurred at 12 weeks after the intervention with FGMS, which confirms that the FGMS intervention significantly lowered management distress among the T1D patients.

Disturbed eating behaviors frequently surface among patients with T1D, with the prevalence among teenagers with T1D reported to be in the range of $30-50 \%[25,26]$. In this patient group, both management and eating distresses have been reported to be major sources of specific frustrations and anxieties related to crucial behavioral demands, such as insufficient blood glucose monitoring and fears that eating restraints seem to be controlling their lives [10]. The results of our study demonstrate that there was a higher degree of distress due to the frustrations and anxieties regarding management and food consumption in the study population at baseline compared to 12 weeks after initiation of the FGMS; this difference was significant ( $p=0.0001)$, thereby confirming that the use of the FGMS significantly minimized the distress related to management and eating behaviors.

Reports from prior studies show that hypoglycemia distress is a crucial, ongoing cause of DRD that involves the patients' lack of confidence in their ability to quickly recognize and competently deal with hypoglycemic symptoms so as to avoid embarrassment and risk to health, particularly when asleep [10]. It is expected that fears related to hypoglycemia are the most common diabetes-related issue among T1D patients. Although patients may experience other diabetes-related stresses and tensions, no other symptom is typically as immediate, challenging, or frightening as perceiving a severe hypoglycemic event. After patients experience a severe episode, they may be traumatized, and this may trigger ongoing anxieties and fears that do not subside [27]. However, results from recent studies demonstrate that the frequent use of FGMS scanning lowers the frequency of hypoglycemic attacks and the related fear, an outcome confirmed in our study [28].

Stress related to providing care for an adolescent exhibiting depressive symptoms has the potential to create even greater pressure in families than the normal stress linked to parental involvement [29]. Further, when parents realize that their adolescent expresses depressive symptoms, they may consider the adolescent to be less capable of managing his/her diabetes and, therefore, the parents themselves may be less willing to accept the responsibilities of diabetes management [29]. However, it is quite difficult to accept support for and collaboration in diabetes management from family, school, and society in general, even when offered, as such offers can appear to be more discouraging than encouraging, which in turn may precipitate distress among friends/family. In our study, we noted that 12 weeks following 
the initiation of the FGMS, a substantial drop in the DDS "friend/family distress" subdomain was evident in the study population. Other studies have shown a key bidirectional connection between T1D distress and glycemic control, which may emphasize the part played by and the significance of diabetes specialists in managing T1D-related distress. In the present study, there was an appreciable reduction in the DDS subdomain "physician distress" at 12 weeks after the initiation of the FGMS in comparison to the baseline $(p=0.0001)$, which confirms that the use of FGMS for glucose monitoring induces a conspicuous reduction in physician distress.

Earlier studies have revealed a noteworthy linear association between the total DDS score and $\mathrm{HbA} 1 \mathrm{C}$, across the entire scale distribution, even for those patients with mild DRD. As such, DRD must be considered across the whole range of potential distress scores, from low to high. However, the significant relationship between DRD and HbA1C is notably dependent on crosssectional findings and does not suggest causality-changes in DRD 'inducing' alterations in the HbA1C or vice versa [30]. Studies have also revealed that DRD and HbA1C co-vary together over time but that alterations in one do not lead to variations in the other, which would imply a causal relationship. These findings suggest the presence of a complex interrelationship between the emotional aspect of diabetes and glycemic control that may actually necessitate the active role of other secondary behavioral or physiological variables. The FGMS is more beneficial than the traditional fingerpricking method due to patient convenience and the comprehensive data it generates. There is evidence that patients scan themselves more frequently when they use the FGMS [20, 28], which produces improved glycemic control, as confirmed by the current outcomes. The present study revealed a positive correlation between HbA1c and DRD level $(r=0.6)$, clearly indicating that the changes in the DRD 'inducing' changes in the HbA1C are reciprocal.

There are a number of limitations to the present study. These include a relatively small sample size, limited number of risk factors assessed, limited social and demographic factors investigated, its single-center design, and the lack of a control group against which the study group could be compared. Additional studies performed on a larger scale are required to overcome these limitations.

\section{CONCLUSION}

Despite the limitations of the present study, we have obtained valuable data on DRD among T1D patients in Saudi Arabia. In conclusion, the results of this prospective study clearly demonstrate that the DDS subdomain scores were reduced after initiation of the FGMS scanning to determine the blood glucose levels. However, further studies are necessary to ascertain if the FGMS system after prolonged and consistent use will provide enhanced results.

\section{ACKNOWLEDGEMENTS}

We thank the participants of the study.

Funding. No funding or sponsorship received for this study. The Rapid Service Fee was funded by Abbott Diabetes Care.

Authorship. All named authors meet the International Committee of Medical Journal Editors (ICMJE) criteria for authorship for this article, take responsibility for the integrity of the work as a whole, and have given their approval for this version to be published.

Authorship Contributions. All authors were involved in the conception and design and/or analysis and interpretation of the data, and all authors were involved in the drafting of the paper and revising it critically for intellectual content.

Disclosures. Ayman A. Al Hayek, Asirvatham A. Robert and Mohamed A. Al Dawish have nothing to disclose.

Compliance with Ethics Guidelines. All procedures followed were in accordance with the Helsinki Declaration of 1964 , as revised in 
2013. The Research and Ethics Committee of Prince Sultan Military Medical City, Riyadh, Saudi Arabia, approved the study protocol. All participants in the study or their parents/caregivers received instructions regarding their roles and provided signed informed consent prior to recruitment.

Data Availability. The datasets generated during and/or analyzed during the current study are available from the corresponding author on reasonable request.

Open Access. This article is licensed under a Creative Commons Attribution-NonCommercial 4.0 International License, which permits any non-commercial use, sharing, adaptation, distribution and reproduction in any medium or format, as long as you give appropriate credit to the original author(s) and the source, provide a link to the Creative Commons licence, and indicate if changes were made. The images or other third party material in this article are included in the article's Creative Commons licence, unless indicated otherwise in a credit line to the material. If material is not included in the article's Creative Commons licence and your intended use is not permitted by statutory regulation or exceeds the permitted use, you will need to obtain permission directly from the copyright holder. To view a copy of this licence, visit http://creativecommons.org/licenses/bync/4.0/.

\section{REFERENCES}

1. Kanungo A. Myths about type 1 diabetes: awareness and education. Indian $\mathrm{J}$ Endocrinol Metab. 2015;19[Suppl 1]:S24-5.

2. Fisher L, Mullan JT, Arean P, et al. Diabetes distress but not clinical depression or depressive symptoms is associated with glycemic control in both crosssectional and longitudinal analyses. Diabetes Care. 2010;33(1):23-8.

3. Fisher L, Hessler DM, Polonsky WH, Mullan J. When is diabetes distress clinically meaningful?: establishing cut points for the Diabetes Distress Scale. Diabetes Care. 2012;35(2):259-64.
4. Snoek FJ, Kersch NY, Eldrup E, et al. Monitoring of individual needs in diabetes (MIND)-2: follow-up data from the cross-national diabetes attitudes, wishes, and needs (DAWN) MIND study. Diabetes Care. 2012;35(11):2128-32.

5. Joensen LE, Tapager I, Willaing I. Diabetes distress in type 1 diabetes-a new measurement fit for purpose. Diabet Med. 2013;30(9):1132-9.

6. Alvarado-Martel D, Ruiz Fernandez MA, Cuadrado Vigaray $M$, et al. Identification of psychological factors associated with adherence to self-care behaviors amongst patients with type 1 diabetes. J Diabetes Res. 2019;2019:6271591.

7. Tanenbaum ML, Gonzalez JS. The influence of diabetes on a clinician-rated assessment of depression in adults with type 1 diabetes. Diabetes Educ. 2012;38(5):695-704.

8. Al Hayek AA, Al Dawish MA. The potential impact of the FreeStyle libre flash glucose monitoring system on mental well-being and treatment satisfaction in patients with type 1 diabetes: a prospective study. Diabetes Ther. 2019;10(4):1239-48.

9. Dennick K, Sturt J, Speight J. What is diabetes distress and how can we measure it? A narrative review and conceptual model. J Diabetes Complicat. 2017;31(5):898-911.

10. Fisher L, Polonsky WH, Hessler DM, et al. Understanding the sources of diabetes distress in adults with type 1 diabetes. J Diabetes Complicat. 2015;29(4):572-7.

11. Rechenberg K, Whittemore R, Grey M. Anxiety in youth with type 1 diabetes. J Pediatr Nurs. 2017;32: 64-71.

12. Bianchi $\mathrm{C}$, Aragona M, Rodia C, et al. Freestyle Libre trend arrows for the management of adults with insulin-treated diabetes: a practical approach. J Diabetes Complicat. 2018;33:6-12.

13. Blum A. System Freestyle Libre glucose monitoring. Clin Diabetes. 2018;36(2):203-4.

14. Sato T, Oshima H, Nakata K, et al. Accuracy of flash glucose monitoring in insulin-treated patients with type 2 diabetes. J Diabetes Investig. 2018;10: 846-50.

15. Vergier J, Samper M, Dalla-Vale F, et al. Evaluation of flash glucose monitoring after long-term use: a pediatric survey. Prim Care Diabetes. 2019;13(1): 63-70.

16. Bailey T, Bode BW, Christiansen MP, Klaff LJ, Alva S. The performance and usability of a factory- 
calibrated flash glucose monitoring system. Diabetes Technol Ther. 2015;17(11):787-94.

17. Bonora B, Maran A, Ciciliot S, Avogaro A, Fadini GP. Head-to-head comparison between flash and continuous glucose monitoring systems in outpatients with type 1 diabetes. J Endocrinol Investig. 2016;39(12):1391-9.

18. Edge J, Acerini C, Campbell F, et al. An alternative sensor-based method for glucose monitoring in children and young people with diabetes. Arch Dis Child. 2017;102(6):543-9.

19. Slattery D, Choudhary P. Clinical use of continuous glucose monitoring in adults with type 1 diabetes. Diabetes Technol Ther. 2017;19(S2):S55-61.

20. Al Hayek AA, Robert AA, Al Dawish MA. Differences of FreeStyle libre flash glucose monitoring system and finger pricks on clinical characteristics and glucose monitoring satisfactions in type 1 diabetes using insulin pump. Clin Med Insights Endocrinol Diabetes. 2019;12:1179551419861102.

21. Kibbey KJ, Speight J, Wong JL, Smith LA, Teede HJ. Diabetes care provision: barriers, enablers and service needs of young adults with type 1 diabetes from a region of social disadvantage. Diabet Med. 2013;30(7):878-84.

22. Poelman SM, Keeling CP, Metelitsa AI. Practical guidelines for managing patients with psoriasis on biologics: an update. J Cutan Med Surg. 2019;23[1 Suppl]:3s-12s.

23. Lansing AH, Berg CA. Adolescent self-regulation as a foundation for chronic illness self-management. J Pediatr Psychol. 2014;39(10):1091-6.
24. Henriquez-Tejo R, Cartes-Velasquez R. Psychosocial impact of type 1 diabetes mellitus in children, adolescents and their families. Literature review. Rev Chil Pediatr. 2018;89(3):391-8.

25. Bachle C, Stahl-Pehe A, Rosenbauer J. Disordered eating and insulin restriction in youths receiving intensified insulin treatment: results from a nationwide population-based study. Int J Eat Disord. $2016 ; 49(2): 191-6$.

26. Peducci E, Mastrorilli C, Falcone S, et al. Disturbed eating behavior in pre-teen and teenage girls and boys with type 1 diabetes. Acta Biomed. 2019;89(4): 490-7.

27. Polonsky WH, Fisher L, Hessler D, Johnson N. Emotional distress in the partners of type 1 diabetes adults: worries about hypoglycemia and other key concerns. Diabetes Technol Ther. 2016;18(5): 292-7.

28. Al Hayek AA, Robert AA, Al Dawish MA. Evaluation of FreeStyle libre flash glucose monitoring system on glycemic control, health-related quality of life, and fear of hypoglycemia in patients with type 1 diabetes. Clin Med Insights Endocrinol Diabetes. 2017;10:1179551417746957.

29. Wu YP, Hilliard ME, Rausch J, Dolan LM, Hood KK. Family involvement with the diabetes regimen in young people: the role of adolescent depressive symptoms. Diabet Med. 2013;30(5):596-602.

30. Johnson B, Eiser C, Young V, Brierley S, Heller S. Prevalence of depression among young people with type 1 diabetes: a systematic review. Diabet Med. 2013;30(2):199-208. 